\title{
The influence of equi-biaxially fabric prestressing on the flexural performance of woven e-glass/polyester reinforced composites
}

\begin{abstract}
The flexural properties of plain-weave woven fabric-reinforced composites have been investigated to clarify the effects of equi-biaxially fabric prestressing on flexural characteristics. The prestressed composite samples were manufactured by applying the symmetrical tension load to both warp and weft yarns prior to matrix curing. The fabricated samples were tested under different fabric orientation angles, i.e. from warp to bias direction. The decline in the flexural properties of the prestressed composite due to matrix creep was checked. From three-point bending tests, the prestressed samples exhibited a maximum increase in the flexural performance, such as the strength and modulus, of $\sim 16 \%$ at a prestressing level of $50 \mathrm{MPa}$ when compared with unprestressed counterparts. The level of improvement in the flexural properties reduced with increasing fabric orientation angle. The creep was induced in the prestressed matrix and subsequent decline in the improved flexural properties was indicated in the prestressed samples. The decline in flexural properties occurred mostly during the short-term creep.
\end{abstract}

Keyword: Polymer-matrix composites; Woven fabric; Flexural properties; Fibre pretension; Residual stress; Creep 